\title{
Molecular Docking Studies on Anticonvulsant Enaminones Inhibiting Voltage-Gated Sodium Channels
}

\author{
Yayin Fang1*, Jamiya Kirkland ${ }^{1}$, Isis J. Amaye ${ }^{2}$, Patrice Jackson-Ayotunde ${ }^{2}$, Matthew George Jr. ${ }^{1^{*}}$ \\ ${ }^{1}$ Department of Biochemistry and Molecular Biology, College of Medicine, Howard University, \\ Washington, DC, USA \\ ${ }^{2}$ Department of Pharmaceutical Sciences, School of Pharmacy and Health Professions, University of Maryland Eastern Shore, \\ Princess Anne, MD, USA \\ Email: *yfang@howard.edu, mgeorge@howard.edu
}

How to cite this paper: Fang, Y., Kirkland, J., Amaye, I.J., Jackson-Ayotunde, P. and George Jr., M. (2019) Molecular Docking Studies on Anticonvulsant Enaminones Inhibiting Voltage-Gated Sodium Channels. Open Journal of Physical Chemistry, 9, 241-257.

https://doi.org/10.4236/ojpc.2019.94015

Received: November 7, 2019

Accepted: November 14, 2019

Published: November 29, 2019

Copyright $\odot 2019$ by author(s) and Scientific Research Publishing Inc. This work is licensed under the Creative Commons Attribution International License (CC BY 4.0).

http://creativecommons.org/licenses/by/4.0/

\begin{abstract}
Epilepsy is described as the most common chronic brain disorder. A typical symptom of epilepsy results in uncontrolled convulsions caused by temporary excessive neuronal discharges. Although several new anticonvulsants have been introduced, some types of seizures have still not been adequately controlled with these new and current therapies. There is an urgent need to develop new anticonvulsant drugs to control the many different types of seizures. Many studies have shown that the epilepsies involve more than one mechanism and therefore may be responsible for the various types of observed seizures. Recently reported studies have shown that a group of newly synthesized $6 \mathrm{~Hz}$ active anticonvulsant fluorinated $\mathrm{N}$-benzamide enaminones exhibited selective inhibitions of voltage-gated sodium $\left(\mathrm{Na}_{\mathrm{v}}\right)$ channels. $\mathrm{Na}_{\mathrm{v}}$ channels are responsible for the initial inward currents during the depolarization phases of the action potential in excitable cells. The activation and opening of $\mathrm{Na}_{\mathrm{v}}$ channels result in the initial phases of action potentials. We hypothesize that there is an essential pharmacophore model for the interactions between these enaminones and the active sites of $\mathrm{Na}_{v}$ channels. The research reported here is focused on molecular docking studies of the interactions that occur between the fluorinated $\mathrm{N}$-benzamide enaminones and the $\mathrm{Na}_{\mathrm{v}}$ channels. These studies may open an avenue for designing anticonvulsant drugs by inhibiting $\mathrm{Na}_{\mathrm{v}}$ channels.
\end{abstract}

\section{Keywords}

Anticonvulsant, Enaminones, Voltage-Gated Sodium Channels, Structure-Based Drug Design, Molecular Docking, 3D QSAR 


\section{Introduction}

Epilepsy is one of the most frequent neurological abnormalities which affect people of all ages. Currently, an estimated 65 million people worldwide live with epilepsy and close to $80 \%$ of them live in low- to middle- income countries. Seventy percent $(70 \%)$ of epilepsy patients could live a quality life without seizures, if more sufficient and efficient diagnoses and treatments were made available to them. However, the costs associated with uncontrolled seizures are seven times higher than the average of all chronic diseases [1]. In the United States alone, the health care costs associated with epilepsy are approximately $\$ 15.5$ billion per year. The burden of epilepsy worldwide is huge; and the need for safer and more effective anticonvulsant drugs is paramount in order to decrease costs for the treatment of epilepsy [2].

Over the years, numerous efforts have been dedicated towards the discovery of new, safe and effective drugs for treating epilepsy. There are several different types of seizures. Most seizures can be categorized as either focal or generalized. Although several new anticonvulsants have been introduced, they are primarily focused on focal seizures. For some types of seizures, such as absence seizure and clonic seizures of generalized seizures, they still have not been adequately controlled with many of these newer and current therapies [3]. Epilepsy is characterized by uncontrolled convulsions and has been known to be caused by excessive temporary neuronal discharges. In view of the broad etiology of the syndrome, much evidence appears to suggest that there is more than one mechanism responsible for the various seizures. Therefore, the need for different therapeutic agents is to be expected. It has been shown that the voltage-gated sodium $\left(\mathrm{Na}_{\mathrm{v}}\right)$ channels are responsible for the initial inward current during the depolarization phase of the action potential in excitable cells [4] [5] [6]. The voltage-gated sodium $\left(\mathrm{Na}_{\mathrm{v}}\right)$ channels are also associated with the reduction of GAB Aergic transmission and excessive glutamatergic neurotransmission which can result in various prominent physiological abnormalities that can lead to the triggering of epileptic seizures [4] [5] [6].

A number of structurally diverse anticonvulsant active enaminone analogues have been synthesized and studied in our laboratory for over two decades. These compounds have displayed a wide range of structural diversity with good to moderate protection against the maximal electroshock seizure (MES) test and the subcutaneous pentylenetetrazol (ScPTZ) test. The enaminone analogues with the central part of $(-\mathrm{HN}-\mathrm{C}=\mathrm{C}-\mathrm{C}=\mathrm{O})$ have been found to share common pharmacophore for their anticonvulsant activities. A reliable 3D QSAR model has been generated by using ligand-based CoMFA and CoMSIA analyses. This model has been used to predict specific structural and electronic futures which is essential in studying active enaminones and their interactions with potential targets [7]-[13]. The initial results from organic synthesis, biological activity tests and ligand-based 3D QSAR studies have shown promise in the development of new anticonvulsant drugs utilizing various enaminones. However, neither the 
mechanism of the anticonvulsant activities nor the molecular target(s) of these active enaminones have been fully determined or known. There is some evidence that the anticonvulsant activities of these enaminones appear to act through two modes of action: inhibition of sodium channels and via a GAB Aergic pathway [7] [8]. Recent electrophysiology studies have shown that some of a group of newly synthesized $6 \mathrm{~Hz}$ active anticonvulsant fluorinated N-benzamide enaminone analogs exhibit inhibition of $\mathrm{Na}_{\mathrm{v}}$ channels [14]. We hypothesize that there is an essential pharmacophore model that best describes the interactions whichoccur between enaminones with active binding site(s) of $\mathrm{Na}_{\mathrm{v}}$ channels. In other words, there appears to be a common mechanism by which the interactions between anticonvulsant enaminones and target $\mathrm{Na}_{\mathrm{v}}$ channels occur. It may be that these enaminones interact with $\mathrm{Na}_{\mathrm{v}}$ channels in the same binding site(s) and in a similar manner.

$\mathrm{Na}_{\mathrm{v}}$ channels are responsible for the initial inward current during the depolarization phase of the action potential in excitable cells [4] [6]. The activation and opening of sodium channel(s) could result in the initial phase of an action potential. There is great interest in gaining a better understanding of the structure and function relationship of $\mathrm{Na}_{\mathrm{v}}$ channels. However, full-length structures of eukaryotic $\mathrm{Na}_{\mathrm{v}}$ channels are hard to obtain, even in low resolution, because of their long and pseudo-repeating nature. In 2017, a complete crystal structure of an open form of $\mathrm{Na}_{\mathrm{v}}$ channels has been determined at high resolution [15]. The crystal structure of a complete $\mathrm{Na}_{\mathrm{v}}$ sodium channel in a full open conformation is derived from a prokaryote [15]. Sequence alignments of eukaryotic $\mathrm{Na}_{\mathrm{v}}$ channels and prokaryotic $\mathrm{Na}_{\mathrm{v}}$ channels have been shown to share only $18 \%$ to $28 \%$ sequence identity [16]. However, some bacterial $\mathrm{Na}_{\mathrm{v}}$ channels have shown similar functions or responses as that of human $\mathrm{Na}_{\mathrm{v}}$ channels. As an example of similar function or response between bacterial and human $\mathrm{Na}_{\mathrm{v}}$ channels, both have been shown to be blocked by human $\mathrm{Na}_{\mathrm{v}}$ channel blockers [16] [17]. Even though the sequence identities are lower than $30 \%$, it appears that function(s) of the $\mathrm{Na}_{\mathrm{v}}$ channels (bacterial and human) have been conserved over evolutionary time and probably share some common structural features as well. The use of this high resolution, full-length $\mathrm{Na}_{\mathrm{v}}$ channel crystal structure will serve as a solid basis or base for enhancing our understanding of the observed inhibition of $\mathrm{Na}_{\mathrm{v}}$ channels by interactions with the enaminones of this study. In taking advantage of the complete crystal structure of the open form $\mathrm{Na}_{\mathrm{v}}$ channel, molecular docking studies will be utilized to determine the active binding site(s) of the enaminones to the $\mathrm{Na}_{\mathrm{v}}$ channels. The molecular docking studies will allow one to gain insight of the interactions that occur between the enaminones and the open form $\mathrm{Na}_{\mathrm{v}}$ channel. These results will be important in furthering one's understanding of the mechanisms of $\mathrm{Na}_{\mathrm{v}}$ channels inhibition by fluorinated $\mathrm{N}$-benzamide enaminones. Additionally, these results could lead to a breakthrough in the current bottleneck which exists in the design and development of novel, safe and effective therapeutic strategies for treating uncontrolled seizures. 


\section{Methods and Material}

\subsection{Hardware and Software}

The computer used for the computational studies was equipped with Microsoft Windows 7 Professional OS and an Intel Xeon $3.40 \mathrm{GHz}$ dual processor with 64.0 GB physical memory. All molecular docking studies were performed with Molecular Operating Environment software (MOE 2018. 0101) [18].

\subsection{Data Setting}

\subsubsection{Ligand Fluorinated N-Benzamide Enaminones}

Table 1 lists 17 newly synthesized fluorinated N-benzamide enaminones, which were reported to show positive activity in acute anti-seizure rodent models and some of them to inhibit the $\mathrm{Na}_{\mathrm{v}}$ channel in the transport protein. All compounds are comprised of a conjugated system with the general form of $(-\mathrm{NH}-\mathrm{C}=\mathrm{C}-\mathrm{C}=\mathrm{O})$ with one or two methyl group on the 5-cyclic position and various fluorinated substitutions on the aromatic ring. The five compounds highlighted as bold in Table 1 have been found to exhibit relatively stronger inhibition of $\mathrm{Na}_{\mathrm{v}}$ channels [14].

\subsubsection{Target Protein $\mathrm{Na}_{\mathrm{v}}$ Channel}

The target protein used for the molecular docking studies described here, is a tetrameric structure of the Wild-Type Open-form Sodium Channel $\mathrm{Na}_{\mathrm{v}}$ MS transport protein found in Magnetococcus marinus. It is a crystal structure of the complete $\mathrm{Na}_{\mathrm{v}}$ prokaryotic sodium channel in a full open conformation. The monomer of the whole structure was taken from the RCSB Protein Data Bank (PDB) with PDB ID as 5HVX and the tetrameric format of the downloaded $\mathrm{Na}_{\mathrm{v}}$ channel was generated by the biomolecular assembly function built in the RCSB Protein Data Bank [15] [19]. This high resolution, full-length crystal structure of the $\mathrm{Na}_{\mathrm{v}}$ channel served as a solid basis for studying interactions between $\mathrm{Na}_{\mathrm{v}}$ channels and its inhibitor enaminones.

\subsection{Molecular Docking}

\subsubsection{Binding Sites Discovery}

The possible active binding sites in the target tetrameric protein $\mathrm{Na}_{\mathrm{v}}$ channel were identified by the Site Finder module built in MOE. The Site Finder module utilizes alpha-shape and discrete-flow methods developed by Edelsbrunner, et al. [20] [21]. This Site Finder module scores and visualizes individual sites by populating them with dummy atoms which serve as probes denoting zones of tight atom packing available for ligand docking. The collection of dummy atoms, which is also called alpha centers or alpha spheres, are classified as either "hydrophobic" or "hydrophilic". This is dependent upon the region of sphere being occupied in the receptor is preferred by what type of the atoms in the pocket [22]. The hydrophilic alpha sphere represents a spot most useful in the formation of hydrogen bonds and the hydrophobic alpha sphere represents a spot that is involved in hydrophobic interactions. After all binding pockets were identified, the Site Finder module ranked the pockets with their Propensity for 
Table 1 . The 17 newly synthesized enaminones shown the inhibition of the voltage-gated sodium channel in the transport protein.

\begin{tabular}{|c|c|c|c|}
\hline Compound ID & All Benzamides & Compound ID & All Benzamides \\
\hline AAA 34 & & IAA 61 & \\
\hline THA 40 & & IAA 42 & \\
\hline THA 36 & & IAA 52 & \\
\hline WGA 34 & & IAA 65 & \\
\hline CBA 80 & & IAA 69 & \\
\hline SGA 33 & & IAB15 & \\
\hline GSA 62 & & IAB19 & \\
\hline GSA 55 & & IABX & \\
\hline TTA 43 & & & \\
\hline
\end{tabular}

Ligand Binding (PLB) score. The PLB score is based upon the amino acid composition of the pocket. The binding pockets can also be ranked by size, which is defined by the number of alpha spheres comprising the pocket(s).

\subsubsection{Docking Procedure}

The docking procedure employed the standard protocol as implemented in MOE 2018.0101 [18]. To perform docking studies, the ligand enaminones were built by using the Builder module in MOE with energy minimization and stored in the MDB format file. The target protein in this study is the tetrameric $\mathrm{Na}_{\mathrm{v}}$ 
channel. The structure of the target protein download from the RCSB Protein Data Bank was protonated by the Protonate 3D module in MOE prior to being set as the receptor. Additionally, the atoms in one of the previously identified possible binding pockets were selected to comprise the target binding site. During the docking procedure, the receptor protein was set as being structurally rigid, while the ligand enaminones were set as being completely flexible. Additional parameters used for the docking procedure included: Placement: Triangle Matcher; Rescoring 1:Longdon dG [18]. Upon binding, the free energy of binding of a ligand from a given pose was estimated using the London dG scoring function. The higher the binding score, the lower the free energy of binding. The resulting poses with higher binding scores are the poses most likely to be preferred by the ligand docked in the pockets. A total of 30 top-score docking poses were constructed and the best scoring complex of the ligand in a specific site was selected for further analysis [23].

\section{Results and Discussion}

$\mathrm{Na}_{\mathrm{v}}$ channels are responsible for the initial inward currents during depolarization phases of the action potential in excitable cells. With the activation and opening of $\mathrm{Na}_{\mathrm{v}}$ channels resulting in the initial phase of the action potential, $\mathrm{Na}_{\mathrm{v}}$ channels play essential roles in excitable tissues [15]. Molecular docking studies were performed using the full-length open-form sodium channel complexed with ligand enaminones, in order to gain a better understanding of the mechanisms associated with $\mathrm{Na}_{\mathrm{v}}$ channel inhibition by fluorinated $\mathrm{N}$-benzamide enaminones. Additionally, a comparative examination of common interaction characteristics between ligand enaminones and target $\mathrm{Na}_{\mathrm{v}}$ channels will also be derived from these molecular docking studies.

\subsection{Structures of Ligand Enaminones}

The newly synthesized $6 \mathrm{~Hz}$ active anticonvulsant fluorinated N-benzamide enaminones were built using the Builder module in MOE and individually energy minimized with an MMFF94x force field. They were then saved to the MDB format files and subsequently docked into selected possible binding pockets in the molecular docking studies reported here.

\subsection{Structure of the Tetrameric $\mathrm{Na}_{\mathrm{v}}$ Channel}

The structure of the $\mathrm{Na}_{\mathrm{v}}$ channel was taken from the Protein Data Bank (PDB id: 5 HVX). Figure 1 reveals that, the full-length open-form sodium channel is a tetramer with four repeating domains or chains. This structure was obtained by using the biomolecule assembly function of the PDB data bank. Figure 1(a) shows a side view of the tetrameric $\mathrm{Na}_{\mathrm{v}}$ channel that is colored to denote the four individual chains; which show the transmembrane pore domains on top and the alpha helical $\mathrm{C}$ terminal domains located under the pore gate. The structure in its fully activated state, with the outward gating charge position is exposed to the 

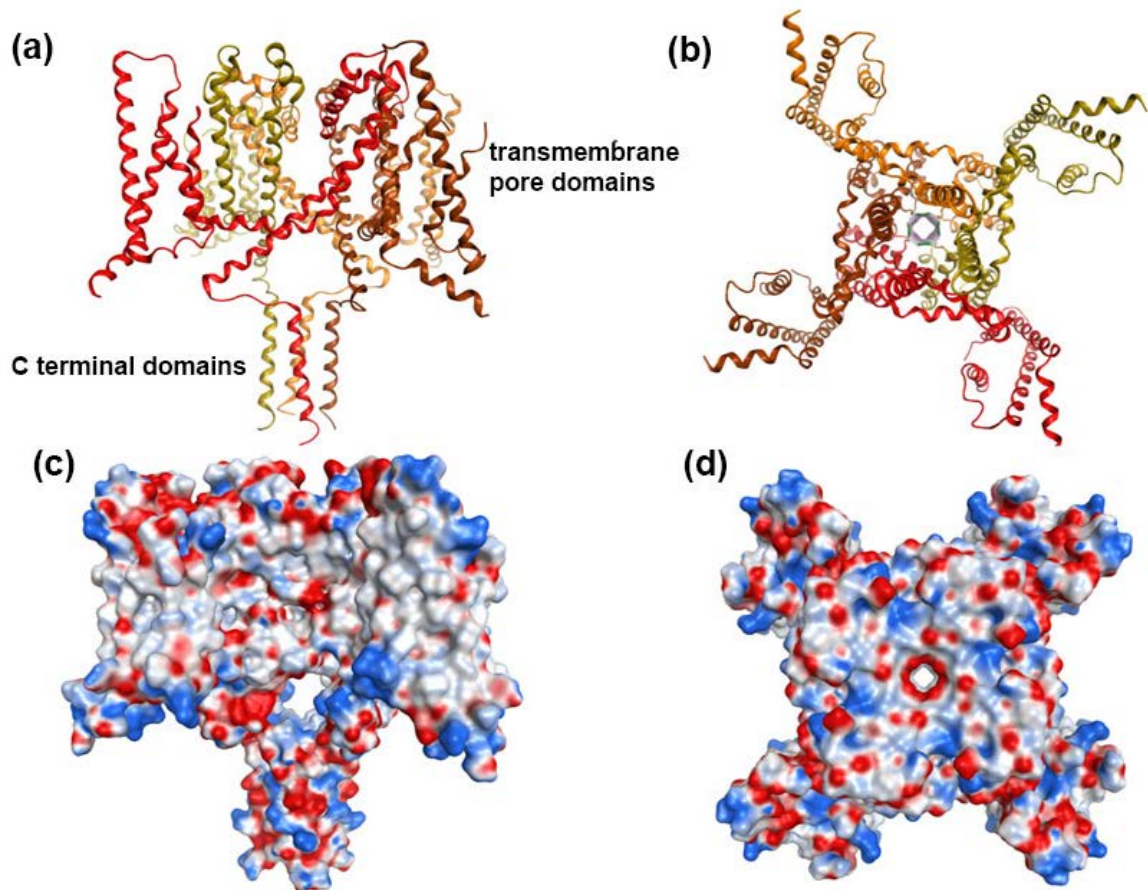

Figure 1. X-Ray crystal structure of the full length wild-type open-form tetrameric $\mathrm{Na}_{\mathrm{v}}$ channel (PDB ID: 5HVX). (a) The side view of the tetrameric $\mathrm{Na}_{\mathrm{v}}$ channel (colored according to monomers), shows the transmembrane pore domains on top and the alpha helical $\mathrm{C}$ terminal domains located under the pore gate. (b) The top view of the tetrameric $\mathrm{Na}_{\mathrm{v}}$ channel from the extracellular surface, shows an open pore gate. (c) Side view of the space filling model of the tetrameric $\mathrm{Na}_{\mathrm{v}}$ channel (colored according to electrostatics, red = negatively charged, blue = positively charged). $(\mathrm{d})$ Top view of the space filling model of the tetrameric $\mathrm{Na}_{\mathrm{v}}$ channel which shows the negatively charged open pore gate, which is sufficiently wide enough to accommodate the passage or flow of sodium ions.

extracellular surface. Figure 1(b) shows the topview of the open pore gate of the tetrameric $\mathrm{Na}_{\mathrm{v}}$ channel from the extracellular surface. Figure $1(\mathrm{c})$ and Figure 1 (d) show the space filling model of the tetrameric $\mathrm{Na}_{\mathrm{v}}$ channel colored according to electrostatics. Sula et al. reported that the negatively charged open pore gate shown in Figure 1(d) is wide enough to accommodate the passage or flow of sodium ions [15].

\subsection{Putative Binding Pocket Discovery}

The MOE binding site analysis described in methods and materials, identified a total of 55 possible binding sites or pockets in the target protein (tetrameric $\mathrm{Na}_{\mathrm{v}}$ channel). Figure 2(a) shows the 55 binding pockets populated with alpha spheres, which are sprayed over the entire target protein. Alpha spheres correspond to regions of the pocket in which the atoms are preferably associated with hydrophilic (red) or hydrophobic (grey) regions of the pocket. The size of a binding pocket is defined by the number of alpha spheres contained within the pocket. Since there were no constraints placed on pocket size during the Site Find procedure, the sizes of the 55 pockets varied from 8 alpha spheres to 340 alpha spheres. Upon inhibition(s) by enaminones of the $\mathrm{Na}_{\mathrm{v}}$ channels [14], a 

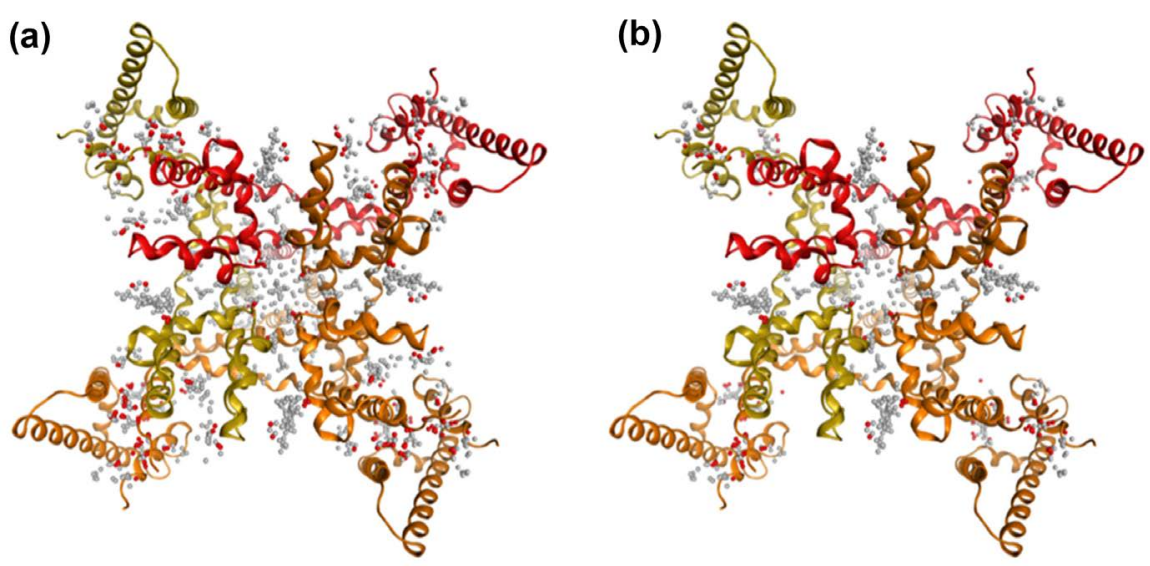

Figure 2. The structure of the tetrameric $\mathrm{Na}_{\mathrm{v}}$ channel highlighted with possible binding pockets. (a). The 55 possible binding pockets populated with alpha spheres in each pocket. Grey and red alpha spheres correspond to hydrophobic and hydrophilicregions in the pockets, respectively. (b). The 15 top sizing possible binding pockets populated with alpha spheres in selected pocket.

good correlation or correspondence should exist or be expected between the sizes of ligand enaminones and active site(s) of the target protein. According to the average molecular weight of the ligand enaminones, a total of 15 top sizing binding sites (containing at least 34 alpha spheres) were chosen as possible binding pockets for the docking studies. Figure 2 (b) shows the alpha spheres located within the 15 chosen pockets, where the alpha spheres were distributed relatively close to the center of the target protein.

Again, recent studies have shown that several newly synthesized anticonvulsant enaminones have exhibited inhibition of $\mathrm{Na}_{\mathrm{v}}$ channels, there is little to no knowledge of where and how these enaminones bind in the active site(s) of the $\mathrm{Na}_{\mathrm{v}}$ channels [14]. Therefore, the docking procedures described in this study were carried out systematically utilizing each of the 17 ligand enaminones and the 15 selected possible binding pockets. During the docking procedure, the target protein was set as being structurally rigid and the ligand was set as being completely flexible. When a ligand was docked in a selected pocket, samples of all possible poses were used to construct binding complex(es). In each given pose, the free energy of binding for the formed complex was estimated by the London dG scoring function implemented in MOE. A total of 30 top-score docking poses were constructed and the best scoring complex for a given ligand in a specific pocket was selected for further analysis. For each ligand, there were 15 top scoring complexes which represented the best pose of the ligand in each specific binding pocket. Based upon molecular docking theory, the pocket which associates with low free energies of binding represents where the ligand prefers to bind. It is expected that the active site or active binding site in a target protein is the pocket where it can generate a ligand-target complex with the highest binding score or lowest free energy of binding. In order to find the active binding site in the tetrameric $\mathrm{Na}_{\mathrm{v}}$ channel, top binding scores of each ligand in the 15 
selected pockets were determined and analyzed. Table 2 lists a comparison of the 15 ranked top scores as exhibited by three representative enaminones. These 3 representative enaminones interact with residues in the binding pockets via hydrogen bonds as determined in the post docking analysis. The top binding scores of all ligands are listed in Supplemental Table S1.

Table 2 and Supplemental Table S1 reveal that every ligand gets its top score when docked into pockets 2, 3, 4 and 5. This analysis indicates that pockets 2 through 5 strongly appear to be the preferred binding pockets for all 17 anticonvulsant enaminones cited in this study. Since Site Finder procedures were carried out on the tetrameric $\mathrm{Na}_{\mathrm{v}}$ channel prior to the docking procedure, the four pockets used for the docking procedures with each ligand were the same. Further investigation found that these four pockets are located in between the chains of the tetramer. In addition, they are identical in size and composition. Each of the four pockets were populated with 88 alpha spheres and comprised of the same group of residues from each of the two chains, between which the pocket is located. Table 3 lists the nine residues and the other six residues from the two chains, respectively.

Figure 3(a) shows the four possible binding pockets located in between the chains of the target tetrameric $\mathrm{Na}_{\mathrm{v}}$ channel. Since the four possible binding pockets reacted the same, results of further analysis will only be focused on pocket 3. Figure 3(b) shows the close-up view of pocket 3 populated with 88 alpha spheres. Alpha spheres serve as guide for the ligand to bind in the pocket.

Table 2. Comparison of ranked binding scores for three representative enaminones.

\begin{tabular}{|c|c|c|c|c|c|}
\hline \multicolumn{2}{|c|}{ IAB15 } & \multicolumn{2}{|c|}{ IAA69 } & \multicolumn{2}{|c|}{ AAA34 } \\
\hline Pocket & Score & Pocket & Score & Pocket & Score \\
\hline 5 & -5.763 & 4 & -6.071 & 5 & -5.616 \\
\hline 2 & -5.755 & 5 & -6.060 & 4 & -5.609 \\
\hline 3 & -5.728 & 3 & -6.049 & 3 & -5.603 \\
\hline 4 & -5.736 & 2 & -6.029 & 2 & -5.584 \\
\hline 8 & -5.211 & 29 & -5.385 & 26 & -5.243 \\
\hline 9 & -5.158 & 6 & -5.335 & 29 & -5.004 \\
\hline 7 & -5.125 & 1 & -5.279 & 6 & -4.800 \\
\hline 6 & -5.121 & 8 & -5.226 & 7 & -4.791 \\
\hline 29 & -5.105 & 7 & -5.225 & 8 & -4.737 \\
\hline 1 & -4.980 & 9 & -5.217 & 9 & -4.742 \\
\hline 26 & -4.957 & 26 & -5.035 & 1 & -4.9613 \\
\hline 14 & -4.663 & 13 & -4.960 & 15 & -4.522 \\
\hline 12 & -4.662 & 12 & -4.935 & 14 & -4.518 \\
\hline 15 & -4.631 & 15 & -4.900 & 13 & -4.473 \\
\hline 13 & -4.626 & 14 & -4.631 & 12 & -4.454 \\
\hline
\end{tabular}


Table 3. Residues contained in each active binding pocket. They are the same in pockets 2 through 5 . Three ligand enaminones interact with target tetrameric $\mathrm{Na}_{\mathrm{v}}$ channel via hydrogen bonds with Lys166 and Tyr169.

\begin{tabular}{cc}
\hline Chain \#1 & Phe142, Glu159, Trp160, Ser165, Lys166, Leu168, Try169, Thr170, Phe172 \\
Chain \#2 & Arg186, Met189, Asn190, Pro193, Trp196, Ile200 \\
\hline
\end{tabular}

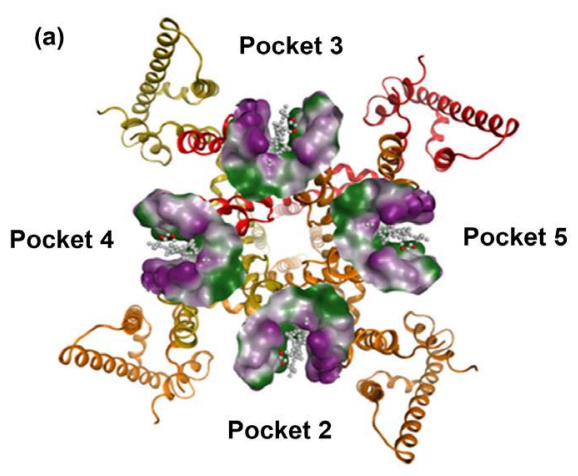

(b)

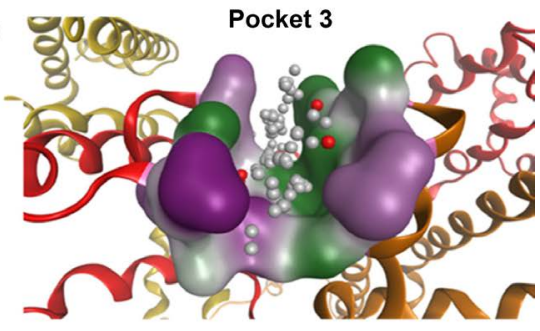

(c)

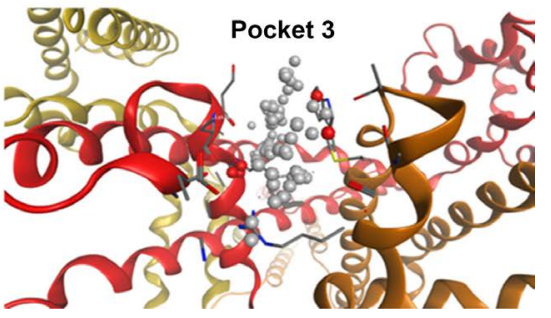

Figure 3. (a) Four identified top scoring pockets from MOE analysis are located in between the chains of the tetrameric $\mathrm{Na}_{\mathrm{v}}$ channel. Each pocket is populated with alpha spheres indicating the type of atoms preferred by the atoms in the pockets (red = hydrophilic, grey $=$ hydrophobic). The solid surfaces representing the lipophilicity surface around the binding pocket with hydrophilic and lipophilic regions highlighted as green and purple, respectively. (b) Shows the close-up view of pocket 3 populated with alpha spheres and (c) Shows the contact residues, along with the local backbone and secondary structure in pocket 3 .

The colors of the alpha spheres indicate the type of atoms preferred by the atoms in the pockets (red = hydrophilic; grey = hydrophobic). The locations of alpha spheres indicate where the atoms with certain type are preferred in the pocket. Among the 88 alpha spheres in Figure 3(b), 82 of them are hydrophobic while six of them are hydrophilic. The location(s) of the hydrophobic spheres are found in the middle and towards the side of the alpha helical $\mathrm{C}$ terminal domains. The alpha helical $\mathrm{C}$ terminal domains are located under the pore gate. The six hydrophilic spheres are found outward of the gating charge position which is exposed to the extracellular surface. The solid surfaces represent lipophilicity of the binding pocket with hydrophilic highlighted in green and lipophilic highlighted in purple regions, respectively. Figure 3 (c) shows the contact residues along with the local backbone and secondary structure of pocket 3 . These contact residues were consistently found in pockets 2,4 and 5 as well as 3 .

\subsection{Post Docking Analysis}

In order to evaluate the possible mechanism(s) of the interactions which may occur between the anticonvulsant enaminones and target $\mathrm{Na}_{\mathrm{v}}$ channel, post 
docking analyses were carried out via the superimposition of all 17 anticonvulsant enaminones docked in pocket 3 .

First, post docking analyses were carried out by examining the top scoring poses of all 17 anticonvulsant enaminones when docked in pocket 3. Figure 4(a) shows the superimposition of all 17 anticonvulsant enaminones docked in pocket 3 . The 16 ligands were found to be deeply bound in the pocket with their structural cores (the cyclic enaminone moiety and benzene ring) fully buried inside. In addition, as indicated by the arrow, Figure 4(a) also shows that ligand IAA61 binds in the pocket differently. By its top scoring pose, the benzene ring of ligand IAA61 extends out of the pocket. Figure 4(b) shows another angle of pocket 3 populated with alpha spheres, which indicates that the binding pocket is a long and narrow groove. Within this long and narrow groove the preferred hydrophobic atoms are packed in the middle and towards the C-terminal domain, while the hydrophilic atoms are located on the side towards the gating charge position that is exposed to the extracellular surface. Figure 4(c) shows that 16 anticonvulsant enaminones docked into pocket 3 with a good respondence between the alpha spheres and the atoms of the ligands. Thei rhydrophobic structural cores are positioned tightly along with the distribution of hydrophobic alpha spheres, while the substituted trifluoromethyl groups of the benzene rings follow the path of the hydrophilic spheres (shown in red). Figure 4(c) also shows that ligand IAA61 docks in the pocket differently. Its benzene ring slides away from the alpha spheres and center of the pocket.

The second approach of post docking analysis consists of a comparative examination of binding cores in pocket 3 of the 17 ligand enaminones. This comparative evaluation reveals how well each ligand binds in the pocket. Figure 5 shows the comparison of binding scores of all ligands docked in pocket 3 . The binding score of different ligands varies. In this study, the binding score is the free energy of binding calculated by the London dG scoring function. A ligand with lower free energy of binding would be more favorable by atoms in the pocket. Figure 5 shows the free energy of binding in the pocket for ligand IAA69 is -6.0597 and has the lowest free energy of binding among the 17 ligands in this study. Therefore, ligand IAA69 is the most favored ligand for pocket 3 . The free energy of binding in pocket 3 for ligand IAA61 is -5.574 , indicating that it would be less favorable for binding in pocket 3 when compared to ligand IAA69.

Figure 6 shows the structural comparison of the binding complex of ligands IAA 69 and IAA 61 in pocket 3. As shown in Figure 5, ligand IAA69 has the lowest free energy of binding among the group, indicating that ligand IAA69 could be more favorable for binding in pocket 3 than other ligands. Figure 6(a) shows a close-up view of ligand IAA69 docked in pocket 3. In the circle highlighted blue in Figure 6(b) reveals a good correspondence between the hydrophobic alpha spheres and the hydrophobic groups of ligand IAA69 are visibly clear. The cyclic enaminone moiety and the benzene ring of ligand IAA69 tightly overlap with the hydrophobic alpha spheres in the pocket. The two substituted 


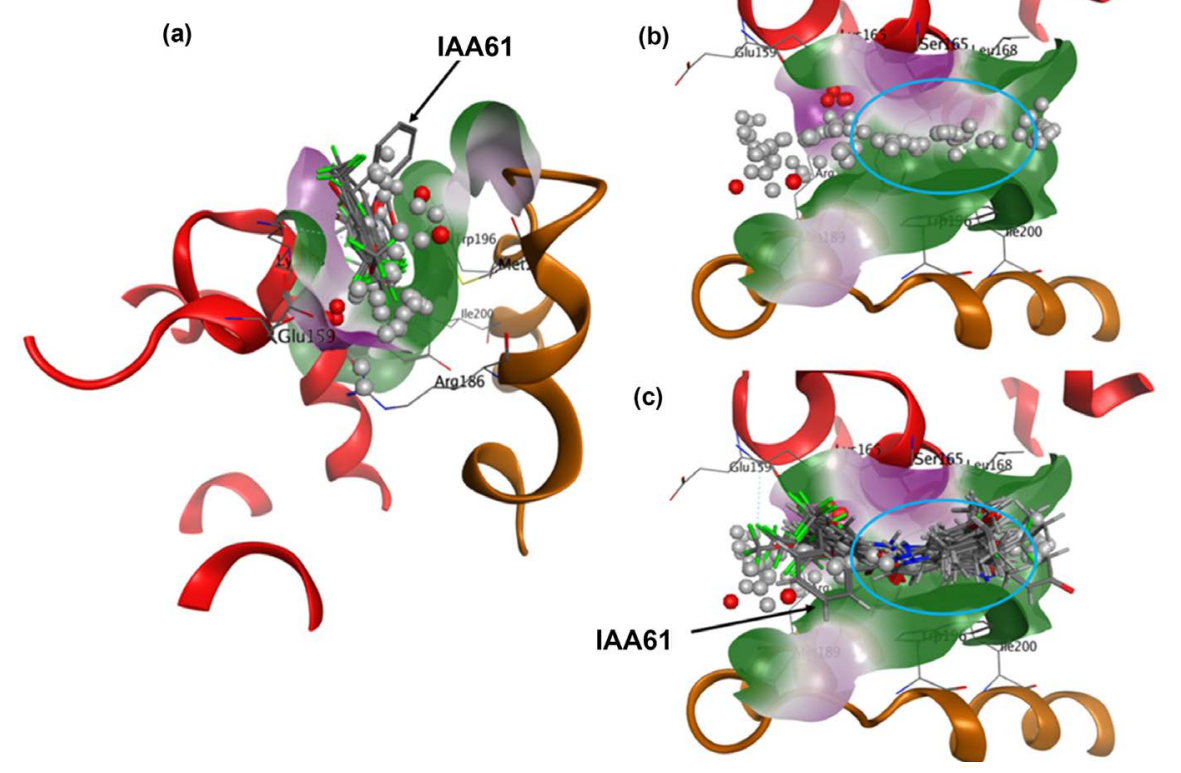

Figure 4. (a) Shows the superimposition all 17 anticonvulsant enaminones docked into pocket 3 with contact residues shown as well. This result is consistent with docking in pockets 2, 4 and 5. (b) Shows the side view of pocket 3 populated with alpha spheres, which indicate the type and position of atoms of ligand enaminones preferred by atoms of contact residues in the pocket. (c) Shows a side view of all 17 anticonvulsant enaminones docked into pocket 3 indicating a good correspondence between the alpha spheres and the atoms of the ligands.

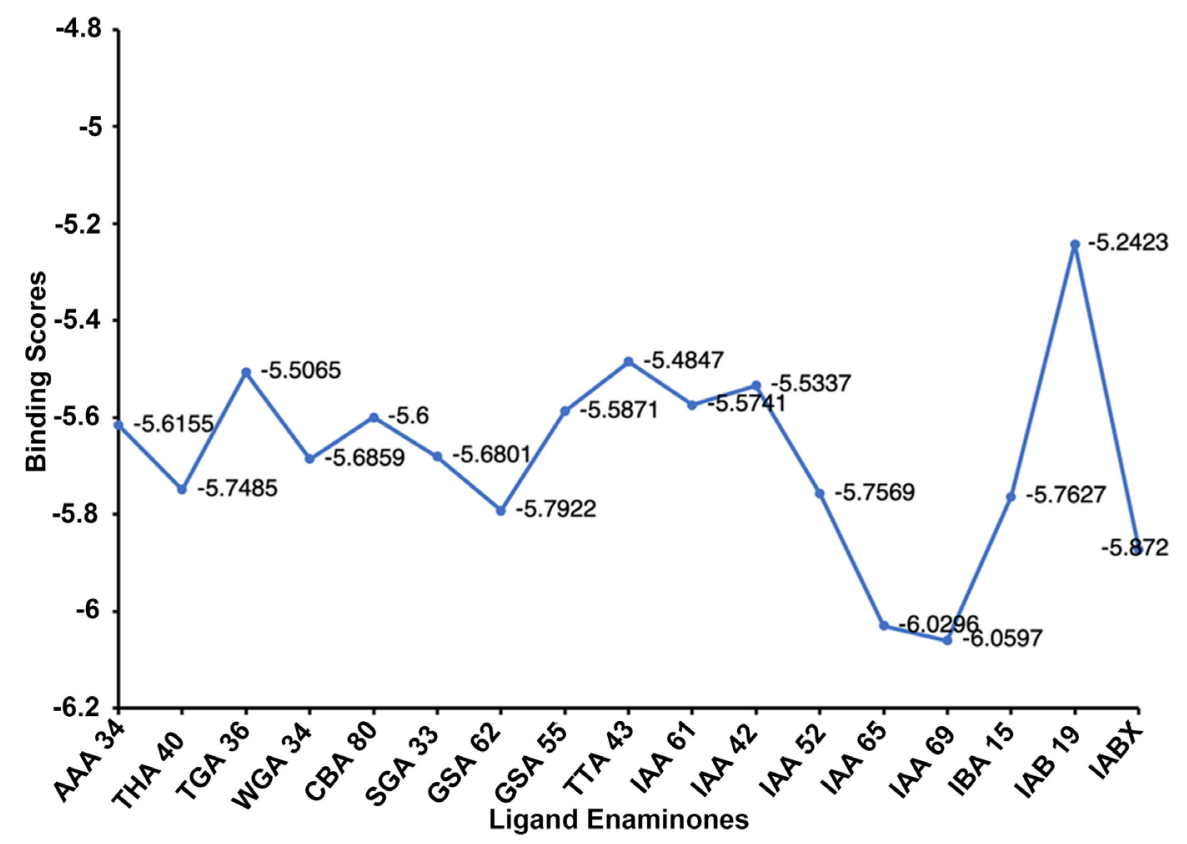

Figure 5. Top binding scores for 17 ligand enaminones docked in Pocket 3.

trifluoromethyl groups on benzene ring are also well matched with the hydrophilic alpha spheres in the pocket. Both Figure 6(a) and Figure 6(b) indicate strong interactions between ligand IAA69 and the target $\mathrm{Na}_{\mathrm{v}}$ channel. In 


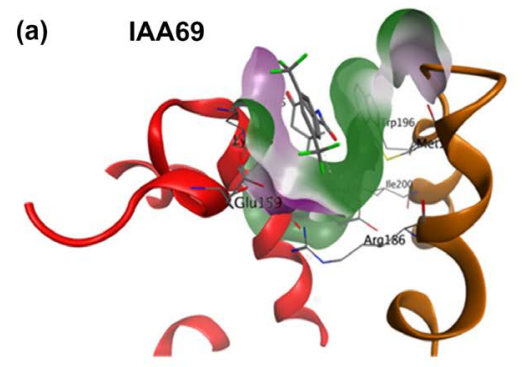

(b)
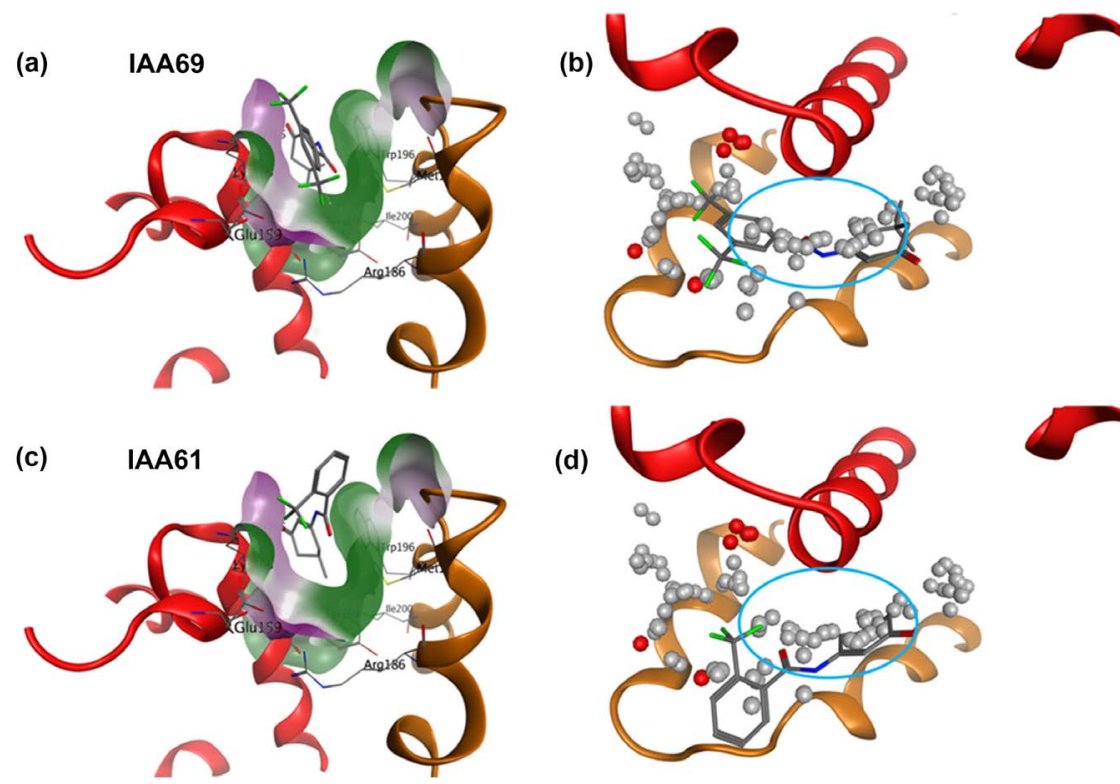

(d)
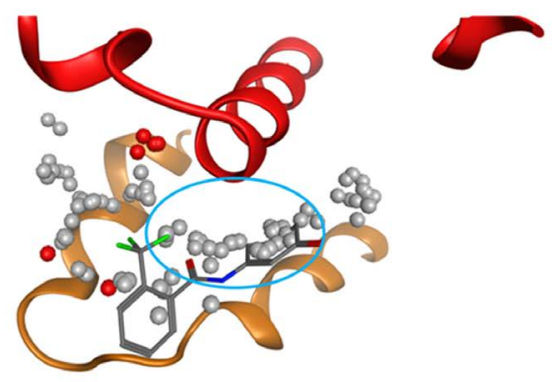

Figure 6. (a) A close-up view of ligand IAA69 docket in pocket 3. (b) A good correspondence between hydrophobic alpha spheres and the hydrophobic groups of ligand IAA69 is clear visible in the circle highlight blue. It shows that the shape of the ligand is overlapped with the collection of alpha spheres. The two trifluoromethyl groups are also well matched with the hydrophilic alpha spheres in the pocket. (c) A close-up view of ligand IAA61 docked in pocket 3 showing that ligand IAA61 is positioned away from the bottom of the pocket 3. (d) Shows the hydrophobic benzene ring of ligand IAA61 is positioned away from the guide of the hydrophobic alpha spheres.

contrast, the binding score of ligand IAA61 appears to be less favorable for binding in pocket 3 than that of ligand IAA69. Figure $6(\mathrm{c})$ shows that ligand IAA61 does not dock deeply in the bottom of pocket 3 . Its benzene ring is contained on the top of the pocket. In addition, as shown within the blue circle of Figure 6(d), the hydrophobic structural core of the ligand is away from the guide/(path) of the hydrophobic alpha spheres. The chemical structure of ligand IAA61 reveals that the benzene ring is monosubstituted with a trifluoromethyl group at the ortho position. The trifluoromethyl group in the ortho position is too bulky to block the free rotations of the substitute on the aromatic ring. As a consequence, the aromatic ring itself would be too bulky to allow the ligand to bind deeply into the pocket. Therefore, the interactions between ligand IAA61 and the target $\mathrm{Na}_{\mathrm{v}}$ channel are not as strong when compared with the interactions of ligand IAA69 and the target $\mathrm{Na}_{\mathrm{v}}$ channel.

Further analysis of the interactions between anticonvulsant enaminones and target $\mathrm{Na}_{v}$ channel is focused on the hydrogen bonds between three ligands (AAA43, IAA69 and IAA15) and two contact residues, Lys166 and Tyr169. Figure $7(\mathrm{a})$ is a close-up view of the three ligands docked in pocket 3. Figure 7(a) reveals that ligands AAA43, IAA69, IAA15 formed hydrogen bonds with the contact residues, Lys166 and Tyr169, in pocket 3. Figures 7(b)-(d) show the 2D diagrams of ligand interaction maps containing contact residues in the pocket and the details of the hydrogen bonds. There are 20,18 and 16 contact residues 

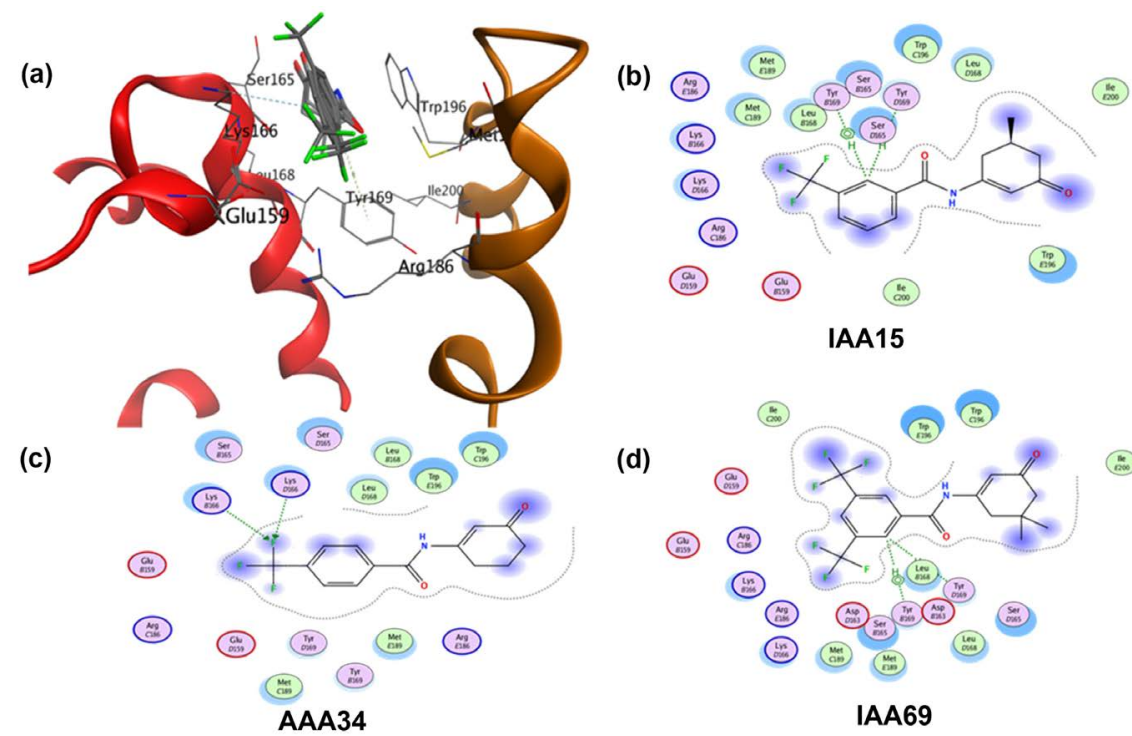

(d)

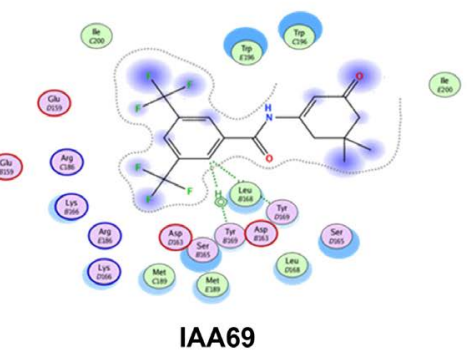

Figure 7. (a) A close-up view of three ligands (AAA34, IAA69 and IAA15) docked in pocket 3, showing important hydrogen bonds between ligands and two contact residues, Lys166 and Tyr169. Figures 7(b)-(d) are 2D diagrams of ligand interaction maps for ligands AAA34, IAA69 and IAA15, respectively. The 2D interaction maps contain contact residues in the pocket and details of the hydrogen bonds.

in the interaction maps of IAA69, IAA15 and AAA34, respectively. It is known that more contact residues in the interaction maps could indicate stronger interactions between ligand and target. For the three ligands, the numbers of contact residues in their $2 \mathrm{D}$ ligand interactions map are correlated with their $\mathrm{Na}_{\mathrm{v}}$ channel inhibition activities, as well as, their free energies of binding. Recall from Table 1, IAA15 is one of the five enaminones that showed strong inhibition of sodium channels in vitro. IAA69 has the lowest free energy of binding as shown in Figure 5. In contrast, the free energy of binding for ligand AAA34 is not as low as that of ligand IAA69 and ligand IAA15.

\section{Conclusion}

Molecular docking studies were carried out on 17 active anticonvulsant enaminones and their interactions with a tetrameric $\mathrm{Na}_{\mathrm{v}}$ channel. The results suggest that there are four identical pockets in the tetrameric $\mathrm{Na}_{\mathrm{v}}$ channel that putatively serve as binding sites for the enaminones that result in the inhibition of $\mathrm{Na}_{\mathrm{v}}$ channels. These four pockets are identical in size and composition and are located in between the four-repeating chains of the $\mathrm{Na}_{\mathrm{v}}$ channel reported here. In this study, it was found that the binding of enaminones and $\mathrm{Na}_{\mathrm{v}}$ Channels resulted in the identification of a set of common features and/or mechanism of interactions between the enaminones and the target $\mathrm{Na}_{\mathrm{v}}$ channel. This was evidenced by the superimposition of docking conformations and by analysis of free energies of binding. The results revealed that all ligand enaminones interacted with contact residues in a given pocket in a similar pattern or mechanism, which was demonstrated by the distribution of alpha spheres and formations of hydro- 
gen bonds with two important residues in the active binding sites. To our knowledge, this is the first structure-based 3D QSAR study on the inhibition of sodium channels by anticonvulsant enaminones. The common features or mechanism of interactions between anticonvulsant enaminones and target $\mathrm{Na}_{\mathrm{v}}$ channels discovered, correlate well with the 3D QSAR model generated by using ligand-based CoMFA and CoMSIA analysis. The discovery of putative binding sites and common features or mechanism of interactions between anticonvulsant enaminones and target $\mathrm{Na}_{\mathrm{v}}$ channels provide a basis for further characterization of pharmacophores and ligand-target binding complexes, which will allow one to design more potent and effective anticonvulsant drugs. Studies such as this will lead to better analysis and selective enhancement of drug candidates for GABAergic disorders such as epilepsy.

\section{Acknowledgements}

This work was supported in part by grants from National Science Foundation (Nos. 1708959 \& 1924092) and Office of Naval Research (Nos. N00014-18-1-2145 \& N00014-17-1-2105) to Yayin Fang, grant and award from American Association of Colleges of Pharmacy and UMES Richard Bernstein Endowed Professorship (respectively) to Patrice Jackson-Ayotunde, and grant from National Institute of Health (No. 2U54MD007597) to RCMI program at the Howard University.

\section{Conflicts of Interest}

The authors declare no conflicts of interest regarding the publication of this paper.

\section{References}

[1] World Health Organization (2019) Epilepsy 2019. https://www.who.int/mental_health/neurology/epilepsy/en/

[2] Citizens United for Research Epilepsy (2019) What Is Epilepsy? http://www.cureepilepsy.org/what-is-epilepsy

[3] French, J.A. and Staley, B.A. (2012) AED Treatment through Different Ages: As Our Brains Change, Should Our Drug Choices Also? Epilepsy Currents, 12, 22-27. https://doi.org/10.5698/1535-7511-12.4s.22

[4] Anger, T., Madge, D.J., Mulla, M. and Riddall, D. (2001) Medicinal Chemistry of Neuronal Voltage-Gated Sodium Channel Blockers. Journal of Medicinal Chemistry, 44, 115-137. https://doi.org/10.1021/jm000155h

[5] Ragavendran, J.V., Sriram, D., Kotapati, S., Stables, J. and Yogeeswari, P. (2008) Newer GABA Derivatives for the Treatment of Epilepsy Including Febrile Seizures: A Bioisosteric Approach. European Journal of Medicinal Chemistry, 43, 2650-2655. https://doi.org/10.1016/j.ejmech.2008.01.036

[6] Wu, T., Ido, K., Ohgoh, M. and Hanada, T. (2019) Mode of Seizure Inhibition by Sodium Channel Blockers, an SV2A Ligand, and an AMPA Receptor Antagonist in a Rat Amygdala Kindling Model. Epilepsy Research, 154, 42-49.

https://doi.org/10.1016/j.eplepsyres.2019.03.011 
[7] Alexander, S.M., Harkless, J., Butcher, J.R., Scott, K.R. and Jackson-Ayotunde, L.P. (2013) Enaminones 11. An Examination of Some Ethyl Ester Enaminone Derivatives as Anticonvulsant Agents. Bioorganic \& Medicinal Chemistry, 21, 3272-3279. https://doi.org/10.1016/j.bmc.2013.03.036

[8] Heinbockel, T., Wang, Z. and Jackson-Ayotunde, P.L. (2014) Allosteric Modulation of GABAA Receptors by an Anilino Enaminone in an Olfactory Center of the Mouse Brain. Pharmaceuticals, 7, 1069-1090. https://doi.org/10.3390/ph7121069

[9] Wang, Z., Sun, L., Jackson, P.L., Scott, R.K. and Heinbockel, T. (2011) A Substituted Anilino Enaminone Acts as a Novel Positive Allosteric Modulator of GABAA Receptors in the Mouse Brain. Journal of Pharmacology and Experimental Therapeutics, 336, 916-924. https://doi.org/10.1124/jpet.110.173740

[10] Jackson, P.L., Hanson, C.D., Farrell, A.K., Raymond, B.J., Stables, J.P., Eddington, N.D. and Scott, K.R. (2012) Enaminones 12. An Explanation of Anticonvulsant Activity and Toxicity Per Linus Pauling's Clathrate Hypothesis. European Journal of Medicinal Chemistry, 51, 42-51. https://doi.org/10.1016/j.ejmech.2012.02.003

[11] Anderson, A.J., Nicholson, J.M., Bakare, O., Butcher, R.J., Wilson, T.L. and Scott, K.R. (2006) Enaminones 9. Further Studies on the Anticonvulsant Activity and Potential Type IV Phosphodiesterase Inhibitory Activity of Substituted Vinylic Benzamides. Bioorganic \& Medicinal Chemistry, 14, 997-1006. https://doi.org/10.1016/j.bmc.2005.09.023

[12] Jackson, P.L., Scott, K.R., Southerland, W.M. and Fang, Y. (2009) Enaminones 8. CoMFA and CoMSIA Studies on Some Anticonvulsant Enaminones. Bioorganic \& Medicinal Chemistry, 17, 133-140. https://doi.org/10.1016/j.bmc.2008.11.014

[13] Edafiogho, I.O., Kombian, S.B., Anathalaskshmi, K.V.V., Salama, N.N., Eddington, N.D., Wilson, T.L., Alexander, M.S., Jackson, P.L., Hanson, C.D. and Scott, K.R. (2007) Enaminones: Exploring Additional Therapeutic Activities. Journal of Pharmaceutical Sciences, 96, 2509-2531. https://doi.org/10.1002/jps.20967

[14] Amaye, I.J., Heinbockel, T., Woods, J., Wang, Z., Martin-Caraballo, M. and Jackson-Ayotunde, P. (2018) $6 \mathrm{~Hz}$ Active Anticonvulsant Fluorinated N-Benzamide Enaminones and Their Inhibitory Neuronal Activity. International Journal of Environmental Research and Public Health, 15, 1784.

https://doi.org/10.3390/ijerph15081784

[15] Sula, A., Booker, J., Ng, L.C., Naylor, C.E., DeCaen, P.G. and Wallace, B.A. (2017) The Complete Structure of an Activated Open Sodium Channel. Nature Communications, 8, 14205-14214. https://doi.org/10.1038/ncomms14205

[16] Bagnéris, C., DeCaen, P.G., Naylor, C.E., Pryde, D.C., Nobeli, I., Clapham, D.E. and Wallace, B.A. (2014) Prokaryotic NavMschannelas a Structural and Functional Model for Eukaryotic Sodium Channel Antagonism. Proceedings of the National Academy of Sciences of the United States of America, 111, 8428-8433. https://doi.org/10.1073/pnas.1406855111

[17] Kuzmenkin, A., Bezanilla, F. and Correa, A.M. (2004) Gating of the Bacterial Sodium Channel, NaChBac, Voltage-Dependent Charge Movement and Gating Currents. The Journal of General Physiology, 124, 349-356. https://doi.org/10.1085/jgp.200409139

[18] Molecular Operating Environment (MOE) (2018) Chemical Computing Group. http://www.chemcomp.com

[19] RCSB Protein Data Bank. https://www.rcsb.org

[20] Edelsbrunner, H. and Mucke, E.P. (1994) Three-Domensional Alpha Shapes. ACM Transactions on Graphics, 13, 43-72. https://doi.org/10.1145/174462.156635 
[21] Edelsbrunner, H. and Shah, N.R. (1996) Incremental Topological Flipping Works for Regular Triangulations. Algorithmica, 15, 223-241. https://doi.org/10.1007/BF01975867

[22] Dal Ben, D., Buccioni, M., Lambertucci, C., Thomas, A. and Volpini, R. (2013) Simulation and Comparative Analysis of Binding Modes of Nucleoside and Non-Nucleoside Agonists at the $\mathrm{A}_{2 \mathrm{~B}}$ Adenosine Receptor. In Silico Pharmacology, 1, 24-38. https://doi.org/10.1186/2193-9616-1-24

[23] Ogindo, C.O., Khraiwesh, M.H., George Jr., M., Brandy, Y., Brandy, N., Gugssa, A., Ashraf, M., Abbas, M., Southerland, W.M., Lee, C.M., Bakare, O. and Fang, Y. (2016) Novel Drug Design for Chagas Disease via Targeting Trypanosoma cruzi Tubulin: Homology Modeling and Binding Pocket Prediction on Trypanosoma cruzi Tubulin Polymerization Inhibition by Naphthoquinone Derivatives. Bioorganic \& Medicinal Chemistry, 24, 3849-3855.

https://doi.org/10.1016/j.bmc.2016.06.031

Supplemental Table S1. The top binding scores of 17 ligands docked in 15 selected binding pockets.

\begin{tabular}{|c|c|c|c|c|c|c|c|c|c|c|c|c|c|c|c|}
\hline & Pocket 1 & Pocket 2 & Pocket 3 & Pocket 4 & Pocket 5 & Pocket 6 & Pocket 7 & Pocket 8 & Pocket 9 & Pocket 12 & Pocket 13 & Pocket 14 & Pocket 15 & Pocket 26 & Pocket 29 \\
\hline Aaa34 & -4.9613 & -5.5840 & -5.6047 & -5.6090 & -5.6155 & -4.7960 & -4.7914 & -4.7370 & -4.7415 & -4.4548 & -4.4735 & -4.5182 & -4.4523 & -5.2435 & -5.0037 \\
\hline CBA80 & -5.2208 & -5.5121 & -5.5312 & -5.6166 & -5.6060 & -5.0619 & -5.0602 & -5.2459 & -5.2453 & -4.7854 & -4.7784 & -4.7826 & -4.7793 & -5.2865 & -5.4850 \\
\hline GSA55 & -4.8728 & -5.6536 & -5.6124 & -5.7109 & -5.5871 & -5.3230 & -5.3261 & -5.4472 & -5.4516 & -4.6343 & -4.5968 & -4.5847 & -4.5840 & -5.3214 & -4.9680 \\
\hline GSA62 & -4.8405 & -5.7695 & -5.7652 & -5.3054 & -5.7922 & -5.1936 & -5.1851 & -5.1655 & -5.1665 & -4.6331 & -4.6813 & -4.6173 & -4.6745 & -4.8998 & -4.8728 \\
\hline IAA42 & -4.9629 & -5.2862 & -5.6212 & -5.6274 & -5.5337 & -5.5169 & -5.5186 & -5.9795 & -5.9853 & -4.6788 & -4.7672 & -4.6711 & -4.7799 & -4.7944 & -5.0020 \\
\hline IAA52 & -4.8511 & -5.6717 & -5.7494 & -5.4459 & -5.7596 & -5.6076 & -5.6052 & -5.5739 & -5.5755 & -4.7396 & -4.7803 & -4.7317 & -4.7919 & -4.6796 & -4.6605 \\
\hline IAA61 & -4.8163 & -5.5397 & -5.5152 & -5.4652 & -5.5741 & -5.4706 & -5.4745 & -5.1329 & -5.1291 & -4.9077 & -4.9216 & -4.9152 & -4.9225 & -4.5262 & -4.6681 \\
\hline IAA65 & -5.0330 & -6.0393 & -6.0593 & -6.0198 & -6.0296 & -5.1148 & -5.2253 & -5.1127 & -5.1244 & -4.7868 & -4.7675 & -4.7623 & -4.8491 & -5.1993 & -4.7975 \\
\hline IAA69 & -5.2786 & -6.0289 & -6.0495 & -6.0714 & -6.0597 & -5.3353 & -5.2253 & -5.2256 & -5.2173 & -4.9348 & -4.9605 & -5.0653 & -4.9000 & -5.0354 & -5.3852 \\
\hline IAB15 & -4.9801 & -5.7555 & -5.7280 & -5.5360 & -5.7627 & -5.1209 & -5.1254 & -5.2107 & -5.1579 & -4.6618 & -4.6262 & -4.6628 & -4.6310 & -4.9571 & -5.1052 \\
\hline IAB19 & -4.5678 & -5.2241 & -5.2096 & -5.2412 & -5.2423 & -4.8775 & -4.8722 & -5.0244 & -5.0222 & -4.4983 & -4.3367 & -4.5046 & -4.3445 & -4.7782 & -4.5306 \\
\hline IABX & -4.9966 & -5.7016 & -5.8577 & -5.8436 & -5.8172 & -5.2241 & -5.2244 & -5.2380 & -5.2381 & -4.6850 & -4.7109 & -4.7317 & -4.6965 & -5.0339 & -5.0124 \\
\hline SGA33 & -5.2328 & -5.6450 & -5.6604 & -5.6799 & -5.6801 & -5.1903 & -5.1851 & -5.4359 & -5.4345 & -4.7942 & -4.9598 & -4.7880 & -4.9507 & -5.3712 & -5.1262 \\
\hline THA36 & -4.7746 & -5.6076 & -5.6146 & -5.6366 & -5.5065 & -5.1263 & -5.2413 & -5.3020 & -5.2995 & -4.4480 & -4.8687 & -4.4410 & -4.8886 & -5.0378 & -5.1476 \\
\hline THA40 & -4.7331 & -5.6999 & -5.6916 & -5.7777 & -5.7485 & -5.0580 & -5.0458 & -5.0540 & -5.0783 & -4.5479 & -4.8435 & -4.5499 & -4.9164 & -4.9087 & -4.7411 \\
\hline THA43 & -5.3206 & -5.3394 & -5.5125 & -5.4940 & -5.4847 & -5.3168 & -5.3201 & -5.3400 & -5.3382 & -4.5746 & -4.9601 & -4.5969 & -4.9511 & -5.4583 & -5.4175 \\
\hline WGA34 & -4.9966 & -5.5630 & -5.5454 & -5.7289 & -5.6859 & -4.9415 & -4.9346 & -4.9439 & -4.9435 & -4.6236 & -4.6433 & -4.6355 & -4.6513 & -5.2754 & -5.2050 \\
\hline
\end{tabular}

